\title{
An Efficient Riemannian Gradient based Algorithm for Max-Cut Problems
}

This paper was downloaded from TechRxiv (https://www.techrxiv.org).

\section{LICENSE}

CC BY 4.0

SUBMISSION DATE / POSTED DATE

28-01-2021 / 03-02-2021

CITATION

Mohades, Mohamad Mahdi; Kahaei, Mohammad Hossein (2021): An Efficient Riemannian Gradient based Algorithm for Max-Cut Problems. TechRxiv. Preprint. https://doi.org/10.36227/techrxiv.13660991.v1

DOI

10.36227/techrxiv.13660991.v1 


\title{
An Efficient Riemannian Gradient based Algorithm for Max-Cut Problems
}

\author{
Mohamad Mahdi Mohades and Mohammad Hossein Kahaei
}

\begin{abstract}
The max-cut problem addresses the problem of finding a cut for a graph that splits the graph into two subsets of vertices so that the number of edges between these two subsets is as large as possible. However, this problem is NP-Hard, which may be solved by suboptimal algorithms. In this paper, we propose a fast and accurate Riemannian optimization algorithm for solving the max-cut problem. To do so, we develop a gradient descent algorithm and prove its convergence. Our simulation results show that the proposed method is extremely efficient on some already-investigated graphs. Specifically, our method is on average 50 times faster than the best well-known techniques with slightly losing the performance, which is on average 0.9729 of the max-cut value of the others.
\end{abstract}

Index Terms-Max-cut problem, manifold optimization, polynomial time, NP-hard.

\section{INTRODUCTION}

$\mathbf{T}$ HE max-cut problem has diverse applications in very-largescaled-integrated (VLSI) circuit design, statistical physics [1], social networks [2], semisupervised learning [3], and biology [4]. This problem can be defined by considering an undirected graph $G=(V, E, \mathbf{A})$, where $V$ denotes the set of vertices, $E$ is the set of edges connecting the vertices in $V$, and $\mathbf{A}$ denotes the adjacency matrix with nonnegative elements. Without loss of generality and for simplicity of presentation, we address the simple graphs which have finitely many vertices and with no loops or multiple edges. A cut for the graph $G$ splits the vertices into two subsets $S$ and $\bar{S}$, where $S \cap \bar{S}=\emptyset$ and $S \cup \bar{S}=V$. Also, the corresponding cut value is calculated as

$$
w(S, \bar{S})=\sum_{\{i, j\} \in E(S, \bar{S})} a_{i j},
$$

where $E(S, \bar{S})$ is the collection of the edges with one endpoint in $S$ and the other in $\bar{S}$, and $a_{i j}$ is the $(i, j)$ th element of the adjacency matrix $A$. Then, the max-cut problem addresses finding a cut with the maximum cut value.

A naive exact solution for the max-cut problem is to calculate the cut value of all $2^{n}$ possible cuts and comparing their corresponding cut values for finding the max-cut, where $n$ is the number of the graph's vertices. This approach demands $\mathcal{O}\left(m 2^{n}\right)$ computations, where $m$ is the number of the corresponding edges, which is in general unaffordable. This exact algorithm can be contemplated as a complete traversal of a binary decision tree in which each branch is obtained by either allocating a vertex to a given set or not. Then, to reduce the computational complexity of this algorithm, the upper and lower bounds of the max-cut are used to limit the traversal of

The authors are with the School of Electrical Engineering, Iran University of Science \& Technology, Tehran 16846-13114, Iran (e-mail: mohamad_mohaddes@elec.iust.ac.ir; kahaei@iust.ac.ir). the decision tree. Such bounds can be obtained by utilizing heuristic functions [5].

To cope with the NP-hardness of the max-cut problem, polynomial-time $\rho$-approximation algorithms may be utilized. A $\rho$-approximation algorithm finds a solution that is at least $\rho$ times the optimal value, where the constant $\rho$ is called the performance guarantee of the algorithm. Also, $\rho$-approximation for randomized algorithms means that the solution is on average $\rho$ times the optimal value. In [6], a 0.5 -approximation algorithm has been presented for the max-cut problem. This algorithm can be simply explained as the random assignment of the set of vertices of the graph to two subsets based on the uniform bipolar distribution. Following this method, some other approximation algorithms have been presented with slight improvement; such as [7] with a performance guarantee of $0.5+0.5 / \Delta$, where $\Delta$ is the maximum degree. In [8], the authors develop a polynomial-time algorithm based on the SDP and randomized rounding approach which achieves 0.878 -approximation of the max-cut problem. Then, the maxcut problem is formulated as

$$
\begin{array}{ll}
\max _{\mathbf{y}} & \frac{1}{2} \sum_{i<j} a_{i j}\left(1-y_{i} y_{j}\right) \\
\text { s.t. } & y_{i} \in\{-1,1\}, \quad i=1, \ldots, n,
\end{array}
$$

where $\mathbf{y}=\left(y_{1}, \cdots, y_{n}\right)$ is a cut for $G$ showing $V_{i}$ belongs to $S$ for $y_{i}=1$ and to $\bar{S}$ for $y_{i}=-1$. However, since (2) is NP-complete, the following semidefinite relaxation is used,

$$
\begin{aligned}
& \max _{\mathbf{x}_{1}, \ldots, \mathbf{x}_{n}} \frac{1}{2} \sum_{i<j} a_{i j}\left(1-\left\langle\mathbf{x}_{i}, \mathbf{x}_{j}\right\rangle\right) \\
& \text { s.t. }\left\|\mathbf{x}_{i}\right\|_{2}=1, \quad i=1, \ldots, n .
\end{aligned}
$$

Then, a randomized rounding (RR) approach is used to map the optimal solution $\left(\mathrm{x}_{1}^{*}, \cdots, \mathrm{x}_{n}^{*}\right)$ resulted from 3 to a cut. To perform the RR, first a random vector $\mathbf{r}$, is chosen with uniform distribution on the unit sphere $\mathrm{S}^{n-1}$. Then, the label $y_{i}$ is estimated as $\operatorname{sign}\left(\left\langle\mathbf{x}_{i}^{*}, \mathbf{r}\right\rangle\right)$.) In [9], it is proved that the method of [8] results in the best polynomial time $\rho$ approximation algorithm for a maximum cut, provided that the unique games conjecture [10] holds. Also, Håstad proved that approximating the max-cut problem with a performance guarantee better than $16 / 17 \simeq 0.941176$ is NP-hard [11].

Apart from the exact and the $\rho$-approximation algorithms for solving the max-cut problem, a set of inexact methods have been also addressed with no performance guarantee. In [12], the authors use the solution of [8] to initialize a local search to improve the max-cut solution, and also investigate some heuristic methods like Metropolis and simulated annealing (SA). The results show that by growing a graph, the SA performs effectively in terms of quality. In [13], the quality 
of the SDP method of [8], random strategy [6], genetic algorithms, combinatorial algorithm [7], and a divide and conquer (D\&C) strategy are investigated. The results show that genetic algorithms give a quite similar solution to that of the random strategy [6], however, with much more running time. Moreover, it is shown that although the combinatorial method performs worse than the SDP, it is much faster. It is also discussed that the D\&C strategy can present a good compromise between the quality and running time.

In [14], a heuristic rank-2 relaxation is developed and the CirCut algorithm is introduced for solving the max-cut problem. In [15], a variable neighborhood search (VNS) method; which is a local search-based metaheuristic method [16], is combined with the path-relinking (PR) method [17]. The resulting method, called VNSPR, shows well performance in either runtime or accuracy. In [18], an efficient implementation is presented for the scatter search (SS) method of [19] with satisfying approximation results. Also, a dynamic convexized (DC) method in [20] performs a local search on a dynamically updated auxiliary function, which shows good performance in solving the max-cut problem.

In this paper, we present a Riemannian Gradient-based algorithm for solving a Max-Cut problem, which we refer to as the RGMC. It is shown how we can formulate a max-cut problem as an unconstrained optimization problem over a Riemannian manifold. Then, we provide an argument for convergence and prove the convergence of the proposed gradient-based algorithm. Using simulations, we show that our method is much faster than the other ones by a slight decrease in the performance. Specifically, on average it is 50 times faster than the best well-known methods and finds a cut with a value of 0.9729 of the max-cut value of the others.

The remaining of the paper is as follows. Section II includes our main results. Simulations are given in Section [III] Section IV concludes the paper and the definitions and proofs are presented in Section $\mathrm{V}$

\section{MAIN RESULTS}

The max-cut problem in 2 can be rewritten as

$$
\begin{array}{ll}
\max _{\mathbf{y}} & \frac{1}{4} \mathbf{y}^{T} \mathbf{L y} \\
\text { s.t. } & y_{i}^{2}=1, \quad i=1, \ldots, n,
\end{array}
$$

where $\mathbf{L}$ denotes the Laplacian of the graph defined as,

$$
\mathbf{L}=\mathbf{D}-\mathbf{A},
$$

with $\mathbf{D}$ being the degree matrix of the graph. It can be shown that (4) is equivalent to the problem:

$$
\max _{\mathbf{x} \in \mathbb{R}^{n}} g(\mathbf{x})=\frac{1}{4} \operatorname{sgn}(\mathbf{x})^{T} \mathbf{L} \operatorname{sgn}(\mathbf{x}),
$$

where $\operatorname{sgn}(\cdot)$ acts elementwise and

$$
\operatorname{sgn}(q)=\left\{\begin{array}{cc}
1 & q \geq 0 \\
-1 & q<0
\end{array} .\right.
$$

Then, for the optimal solutions of (4) and (6), respectively shown by $\mathbf{y}^{*}$ and $\mathbf{x}^{*}$, we get $\mathbf{y}^{*}=\operatorname{sgn}\left(\mathbf{x}^{*}\right)$. In this way, we have formulated the max-cut problem as a nonsmooth optimization problem over the Euclidean space.

To make (6) differentiable, we approximate $\operatorname{sgn}(q)$ by the differentiable function $\frac{2}{\pi} \tan ^{-1}(\alpha q)$, which becomes more accurate as $\alpha$ increases. Then, (6) can be presented as

$$
g(\mathbf{x}) \simeq \frac{1}{\pi^{2}} \tan ^{-1}(\alpha \mathbf{x})^{T} \mathbf{L} \tan ^{-1}(\alpha \mathbf{x})
$$

Moreover, to incorporate the approximation accuracy in the optimization problem, we use the following penalty term:

$$
\left|(\operatorname{sgn}(q))^{2}-\left(\frac{2}{\pi} \tan ^{-1}(\alpha q)\right)^{2}\right|=\left|1-\left(\frac{2}{\pi} \tan ^{-1}(\alpha q)\right)^{2}\right| .
$$

In this way, we propose the following maximization problem for the max-cut problem,

$$
\begin{aligned}
\max _{\mathbf{x} \in \mathbb{R}^{n}, \alpha \in \mathbb{R}} h(\mathbf{x}, \alpha)= & \frac{1}{\pi^{2}} \tan ^{-1}(\alpha \mathbf{x})^{T} \mathbf{L t a n}^{-1}(\alpha \mathbf{x})- \\
& \sum_{i} \gamma_{i}\left|1-\left(\frac{2}{\pi} \tan ^{-1}\left(\alpha x_{i}\right)\right)^{2}\right|,
\end{aligned}
$$

where $\gamma_{i}$ 's are positive regularization parameters and $x_{i}$ is the $i$ th element of $\mathbf{x}$. Then, the max-cut solution is estimated as $\mathbf{y}^{*}=\operatorname{sgn}\left(\mathbf{x}^{*}\right)$, where $\left(\mathbf{x}^{*}, \alpha^{*}\right)$ is the local optimum of $(8)$. Since this solution is independent of $\alpha$, we can rewrite (8) as,

$$
\begin{array}{r}
\max _{\mathbf{z} \in \mathbb{R}^{n}} h(\mathbf{z})=\frac{1}{\pi^{2}} \tan ^{-1}(\mathbf{z})^{T} \mathbf{L t a n}^{-1}(\mathbf{z})- \\
\sum_{i} \gamma_{i}\left|1-\left(\frac{2}{\pi} \tan ^{-1}\left(z_{i}\right)\right)^{2}\right|,
\end{array}
$$

where $\mathbf{z}^{*}$ is a local optimum solution for 9 , and $\mathbf{y}^{*}=$ $\operatorname{sgn}\left(\mathbf{z}^{*}\right)$.

Next, by expanding (9), we can rewrite it as

$$
\begin{aligned}
\min _{\mathbf{z} \in \mathbb{R}^{n}} \quad-f(\mathbf{z}) & = \\
& -\frac{1}{\pi^{2}} \tan ^{-1}(\mathbf{z})^{T}(\mathbf{L}+4 \operatorname{diag}(\gamma)) \tan ^{-1}(\mathbf{z})+\sum_{i} \gamma_{i},
\end{aligned}
$$

where $\gamma$ is the vector of $\gamma_{i}$ 's and $\operatorname{diag}(\cdot)$ transfers the input vector to a diagonal matrix. To optimize the regularization parameters, we also incorporate $\gamma$ in the problem as

$$
\begin{array}{rl}
\min _{\mathbf{z} \in \mathbb{R}^{n}, \boldsymbol{\gamma} \in \mathbb{R}_{+}^{n}} & F(\mathbf{z}, \boldsymbol{\gamma})= \\
& -\frac{1}{\pi^{2}} \tan ^{-1}(\mathbf{z})^{T}(\mathbf{L}+4 \operatorname{diag}(\gamma)) \tan ^{-1}(\mathbf{z}) \\
& +\sum_{i} \gamma_{i},
\end{array}
$$

where $\mathbb{R}_{+}$is the set of positive real numbers. To solve [11, we propose a gradient descent method over the product manifold $\mathcal{M}=\mathbb{R}^{n} \times \mathbb{R}_{+}^{n}$, as presented in Alg. 1 . Then, the max-cut solution is,

$$
\mathbf{y}^{*}=\operatorname{sgn}\left(\mathbf{z}^{*}\right)
$$

where $\mathbf{X}^{*}=\left(\mathbf{z}^{*}, \gamma^{*}\right) \in \mathcal{M}$ denotes the optimum value obtained from Alg. 1 [21]. 


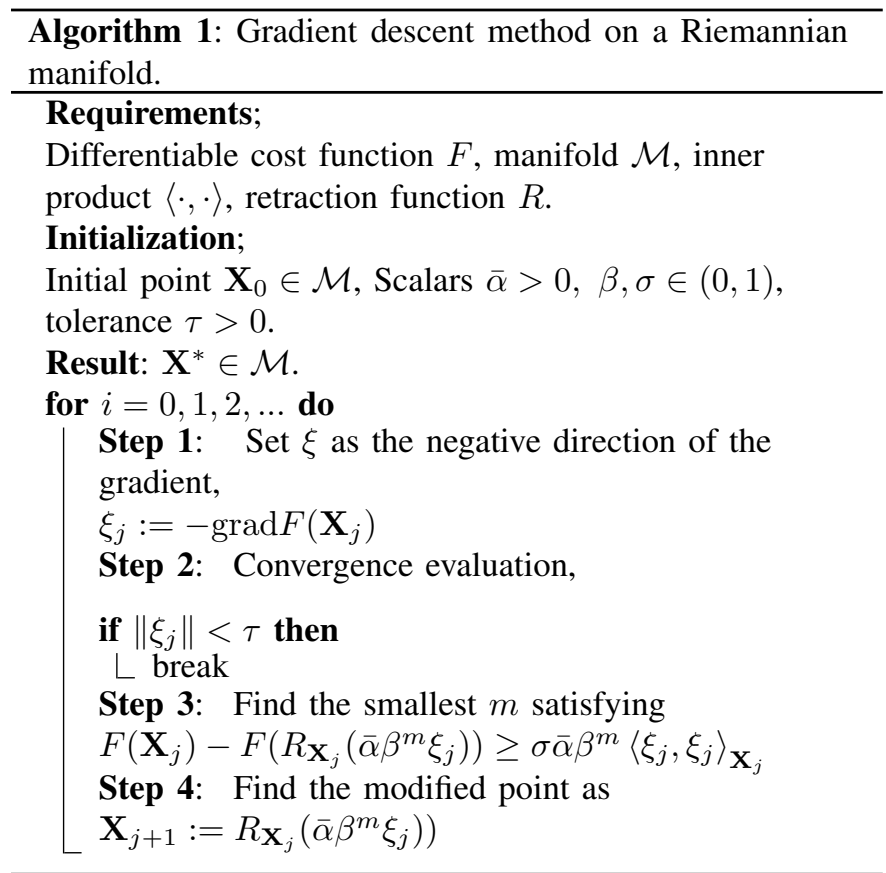

The convergence of Alg. 1 for the problem defined by (11) is investigated in the following.

Lemma 1. A sequence generated by Alg. 1 for (11) does not necessarily converge to a point in $\mathcal{M}=\mathbb{R}^{n} \times \mathbb{R}_{+}^{n}$.

Proof. See Appendix V-B.

To guarantee convergence of Alg. 1, we modify problem (11) so that any sequence generated by Alg. 1 lies in a compact set. After that, we present a theorem for convergence of Alg. 1. To realize the aforementioned compactness, we should create a set that is bounded and closed. For this purpose, we modify problem (11) in two steps. In the first step, we impose the following constraints to (11), which make the feasible set bounded,

$$
\left\{\begin{array}{c}
\|\mathbf{z}\|_{2}=1 \\
\sum_{i} \gamma_{i}=1
\end{array}\right.
$$

where $\|\cdot\|_{2}$ is the $l_{2}$-norm. Then, 11] turns into

$$
\begin{aligned}
\min _{\mathbf{z} \in S^{n-1}, \boldsymbol{\gamma} \in S_{n-1}} F(\mathbf{z}, \boldsymbol{\gamma})= \\
-\frac{1}{\pi^{2}} \tan ^{-1}(\mathbf{z})^{T}(\mathbf{L}+4 \operatorname{diag}(\boldsymbol{\gamma})) \tan ^{-1}(\mathbf{z}),
\end{aligned}
$$

where $S^{n-1}$ is an $(n-1)$-dimensional sphere and $S_{n-1}$ is an $(n-1)$-dimensional statistical manifold (See Definition 5). In the second step, we use the logarithmic-barrier function to confine $\gamma$ within a compact set. Thus, we modify (13) as

$$
\begin{array}{rl}
\min _{\mathbf{z} \in S^{n-1}, \boldsymbol{\gamma} \in S_{n-1}} & H(\mathbf{z}, \gamma)= \\
-\frac{1}{\pi^{2}} \tan ^{-1}(\mathbf{z})^{T}(\mathbf{L}+4 \operatorname{diag}(\gamma)) \tan ^{-1}(\mathbf{z}) \\
-v \sum_{i} \log \left(\gamma_{i}-\varepsilon\right),
\end{array}
$$

where $v$ is an infinitesimal positive value and $\varepsilon$ is a very small value in the interval $(0,1)$. By this construction, any sequence generated by Alg. 1 for (14) will belong to the compact set $S^{n-1} \times\left[\begin{array}{ll}\varepsilon & 1-\varepsilon\end{array}\right]^{n-1}$. However, since $\gamma_{i}=\varepsilon$ is not in the feasible set of [14], we present the following theorem to derive a guarantee for the convergence of (13).

Theorem 1. Let $v$ be an infinitesimal positive value and $\varepsilon$ be a very small value in the interval $(0,1)$ for $(14)$. Also, assume that Alg. 1 generates the infinite sequence $\left\{\mathbf{X}_{j}\right\}_{j=0}^{j \rightarrow \infty}$ for 14. Then, the sequence $\left\{\mathbf{X}_{j}\right\}_{j=0}^{j \rightarrow \infty}$ will have a convergent point, namely $\mathbf{X}_{*} \in \mathcal{N}=S^{n-1} \times S_{n-1}$, for problem $(13$.

Proof. See Appendix V-C

\section{Simulation Results}

To evaluate the performance of the proposed algorithm, we consider four other approaches for comparison purposes, including the SS, CirCut, VNSPR, and DC. Our criteria for evaluation of the methods are the value of max-cut solution as well as the runtime. All the experiments are performed in MATLAB 2015 on a computer with Intel(R) Core(TM) i5$4210 \mathrm{U}$ CPU $(1.7 \mathrm{GHz})$ and $6 \mathrm{~GB}$ RAM.

In the first experiment, we compare the methods over the graph tests G1, G2, G3, G4, G5, G12, G13, G14, G15, G16, G17, G22, G23, G24, G25, G26, G35, G36, G37, G38, G44, G45, G46, G47, G48, G49, G50, G51, G52, G53, G54, which have binary weights generated by Helmberg et al. [22]. The results presented in Table I show that our algorithm is more fast and accurate by finding a cut value with the average ratio of 0.9729 to the best cut and an average runtime ratio of 0.02 to the best of the other algorithms. To initialize the algorithm for solving (14), we apply the singular value decomposition (SVD) to the Laplacian matrix of the graph and choose a singular vector for initializing $\mathbf{z}$ over $S^{n-1}$. We also generate a random point over $S_{n-1}$ to initialize $\gamma$.

\section{CONCLUSION}

We proposed an efficient Riemannian optimization algorithm for the max cut problem. To solve this problem, a Riemannian gradient descent was developed and its convergence was proved. Simulation results showed high accuracy and speed of the proposed algorithm in terms of the cut value and the runtime. Specifically, by inspecting the runtimes, it was concluded that our method is almost 50 times faster than some well-known algorithms at the cost of slightly losing the max-cut value, which is 0.9729 of the others.

\section{APPENDICES}

\section{A. Some Definitions}

In the following some definitions required for Alg. 1 are presented. More information may be found in [21].

Definition 1. Consider the manifold $\mathcal{M}$. The set of all tangent vectors at a point $\mathbf{x} \in \mathcal{M}$ denoted by $T_{\mathbf{x}} \mathcal{M}$ is called the tangent space at this point. Also, the disjoint union of tangent spaces is called the tangent bundle denoted by $T \mathcal{M}$.

Definition 2. Let $\mathcal{M}$ be a manifold. A smooth function, which assigns a tangent vector to each point of $\mathcal{M}$ is called a tangent vector field $\xi$ on $\mathcal{M}$. An example of the tangent vector field 
TABLE I

COMPARISON OF DIFFERENT MAX-CUT ALGORITHMS.

\begin{tabular}{|c|c|c|c|c|c|c|c|c|c|c|c|c|c|c|}
\hline \multicolumn{3}{|c|}{ Instance } & \multicolumn{2}{|c|}{ SS } & \multicolumn{2}{|c|}{ circuit } & \multicolumn{2}{|c|}{ VNSPR } & \multicolumn{2}{|c|}{ DC } & \multicolumn{2}{|c|}{ RGMC } & \multicolumn{2}{|c|}{ Ratio to Best } \\
\hline Name & $|\mathrm{V}|$ & $|\mathrm{E}|$ & value & time & value & time & value & time & value & time & value & time & value & time \\
\hline G1 & 800 & 19176 & 11624 & 139 & 11624 & 352 & 11621 & 22732 & 11624 & 184.422 & 11310 & 1.68 & 0.973 & 0.012 \\
\hline G2 & 800 & 19176 & 11620 & 167.2 & 11617 & 283 & 11615 & 22719 & 11620 & 183.938 & 11304 & 2.22 & 0.973 & 0.013 \\
\hline G3 & 800 & 19176 & 11622 & 180.1 & 11622 & 330 & 11622 & 23890 & 11622 & 183.094 & 11313 & 1.17 & 0.973 & 0.006 \\
\hline G4 & 800 & 19176 & 11646 & 194.4 & 11641 & 523.6 & 11600 & 24050 & 11646 & 187.297 & 11293 & 3.53 & 0.97 & 0.019 \\
\hline G5 & 800 & 19176 & 11631 & 205.2 & 11627 & 1128 & 11598 & 23134 & 11631 & 184.391 & 11300 & 1.54 & 0.972 & 0.008 \\
\hline G14 & 800 & 4649 & 3060 & 186.5 & 3058 & 128 & 3055 & 16734 & 3057 & 115.75 & 2969 & 2.81 & 0.97 & 0.024 \\
\hline G15 & 800 & 4661 & 3049 & 142.8 & 3049 & 155 & 3043 & 17184 & 3044 & 115.407 & 2959 & 7.76 & 0.97 & 0.067 \\
\hline G16 & 800 & 4672 & 3045 & 161.9 & 3045 & 142 & 3043 & 16562 & 3052 & 113.563 & 2958 & 7.3 & 0.969 & 0.064 \\
\hline G17 & 800 & 4667 & 3043 & 312.8 & 3037 & 365.5 & 3030 & 18555 & 3043 & 114.25 & 2951 & 3.53 & 0.97 & 0.031 \\
\hline G22 & 2000 & 19990 & 13346 & 1336 & 13346 & 493 & 13295 & 197654 & 13339 & 957.14 & 12941 & 6.87 & 0.97 & 0.014 \\
\hline G23 & 2000 & 19990 & 13317 & 1022 & 13317 & 457 & 13290 & 193707 & 13323 & \begin{tabular}{|l|}
987.328 \\
\end{tabular} & 12958 & 6.8 & 0.973 & 0.015 \\
\hline G24 & 2000 & 19990 & 13303 & 1191 & 13314 & 521 & 13276 & 195749 & 13314 & \begin{tabular}{|l|}
968.891 \\
\end{tabular} & 12931 & 6.84 & 0.971 & 0.013 \\
\hline G25 & 2000 & 19990 & 13320 & 1299 & 13326 & 1600 & 12298 & 212563 & 13324 & \begin{tabular}{|l|}
972.625 \\
\end{tabular} & 12973 & 9.16 & 0.974 & 0.009 \\
\hline G26 & 2000 & 19990 & 13294 & 1415 & 13314 & 1569 & 12290 & 228969 & 13313 & 965.453 & 12950 & 7.33 & 0.973 & 0.008 \\
\hline G35 & 2000 & 11778 & 7668 & 1258 & 7670 & 440 & 7635 & 167221 & 7647 & 860.312 & 7393 & 7 & 0.964 & 0.016 \\
\hline G36 & 2000 & 11766 & 7660 & 1392 & 7660 & 400 & 7632 & 167203 & 7625 & 858.406 & 7353 & 8.44 & 0.96 & 0.021 \\
\hline G37 & 2000 & 11785 & 7664 & 1387 & 7666 & 382 & 7643 & 170786 & 7640 & 873.203 & 7368 & 13.07 & 0.961 & 0.034 \\
\hline G38 & 2000 & 11779 & 7681 & 1012 & 7646 & 1189 & 7602 & 178570 & 7641 & 886.125 & 7372 & 15.25 & 0.96 & 0.017 \\
\hline G44 & 1000 & 9990 & 6648 & 355.9 & 6643 & 192 & 6642 & 34519 & 6650 & 218.172 & 6481 & 3.31 & 0.975 & 0.017 \\
\hline G45 & 1000 & 9990 & 6642 & 354.3 & 6652 & 210 & 6646 & 34179 & 6647 & 217.391 & 6454 & 2.36 & 0.97 & 0.011 \\
\hline G46 & 1000 & 9990 & 6634 & 498.1 & 6645 & 638.7 & 6630 & 38854 & 6647 & 219.828 & 6452 & 3.38 & 0.971 & 0.015 \\
\hline G47 & 1000 & 9990 & 6649 & 359.1 & 6656 & 632.9 & 6640 & 36587 & 6657 & 220.937 & 6454 & 3.48 & 0.97 & 0.016 \\
\hline G48 & 3000 & 6000 & 6000 & 20.1 & 6000 & 119 & 6000 & 64713 & 6000 & 588.203 & 6000 & 0 & 1 & 0 \\
\hline G49 & 3000 & 6000 & 6000 & 35.1 & 6000 & 134 & 6000 & 64749 & 6000 & 616.359 & 6000 & 0 & 1 & 0 \\
\hline G50 & 3000 & 6000 & 5880 & 26.8 & 5880 & 231 & 5880 & 147132 & 5880 & 773.515 & 5880 & 0 & 1 & 0 \\
\hline G51 & 1000 & 5909 & 3846 & 513 & 3837 & 497.3 & 3808 & 89966 & 3842 & 192.578 & 3735 & 6.12 & 0.971 & 0.032 \\
\hline G52 & 1000 & 5916 & 3849 & 550.8 & 3833 & 506.8 & 3816 & 95985 & 3840 & 185.765 & 3732 & 6.6 & 0.97 & 0.036 \\
\hline G53 & 1000 & 5914 & 3846 & 423.8 & 3842 & 502.5 & 3802 & 92459 & 3844 & 190.75 & 3729 & 11.51 & 0.97 & 0.06 \\
\hline G54 & 1000 & 5916 & 3846 & 429 & 3842 & 523.6 & 3820 & 98458 & 3831 & 190.453 & 3731 & 2.6 & 0.97 & 0.014 \\
\hline
\end{tabular}

is the gradient of a real-valued smooth function $f$ over $\mathcal{M}$, which is denoted by $\operatorname{grad} f$.

Definition 3. A Riemannian manifold is a manifold equipped with a smoothly varying inner product. The inner product over a manifold is denoted by $\langle\cdot, \cdot\rangle$ and its restriction to the tangent space $T_{\mathbf{x}} \mathcal{M}$ is denoted by $\langle\cdot, \cdot\rangle_{\mathbf{x}} \cdot$

Definition 4. Let $\mathcal{M}$ be a Riemannian manifold with tangent bundle $T \mathcal{M}$. The exponential map denoted by $\operatorname{Exp}(\cdot)$ maps the elements of $T \mathcal{M}$ to $\mathcal{M}$, so that for $v \in T \mathcal{M}, \operatorname{Exp}(\mathrm{v})$ equals to $h$ at time 1 ; where $h$ is a unique geodesic starting from the base point of $v$ with velocity $v$ at time 0 . Moreover, the retraction function brings a point on tangent bundle back to the manifold which satisfies some properties mentioned in Definition 4.1.1 of [21]. The first-order approximation of the exponential map is a way of constructing the retraction function.

Definition 5. Let $S_{n}$ be the set of all positive measures:

$$
S_{n}=\left\{\mathbf{p}=\left(p_{0}, \ldots, p_{n-1}\right) \mid 0<p_{i}, i=0, \ldots, n-1\right\} .
$$

Then, the subspace $S_{n-1}$ of $S_{n}$ with the property $\sum_{i=0}^{n-1} p_{i}=1$ is called the statistical manifold.

\section{B. Proof of Lemma 1}

Proof. Let $\left\{\mathbf{X}_{j}\right\}_{j=0}^{j \rightarrow \infty}$ be a sequence generated by Alg. 1 for (11), where $\mathbf{X}_{j}=\left(\mathbf{z}_{j}, \gamma_{j}\right) \in \mathcal{M}$. Also, let $\mathbf{X}^{*}$ be the limit point of the sequence $\left\{\mathbf{X}_{j}\right\}_{j=0}^{j \rightarrow \infty}$ and let the subsequence $\left\{\mathbf{X}_{j}\right\}_{j=N}^{j \rightarrow \infty}$ belong to $B_{\epsilon}\left(\mathbf{X}^{*}\right)$, which is defined as the $l_{2}$-norm ball of radius $\epsilon$ around $\mathbf{X}^{*}$. Now, we prove by contradiction that $\mathbf{X}^{*}$ does not necessarily belong to $\mathcal{M}$. Assume that 
$\mathbf{X}^{*} \in \mathcal{M}$ is a local optimal point for (11). In other words, there exists an arbitrarily small $\rho$-neighbourhood $B_{\rho}\left(\mathbf{X}^{*}\right)$, for which we can write,

$$
F\left(\mathbf{z}_{*}, \boldsymbol{\gamma}_{*}\right) \leq F(\mathbf{z}, \gamma), \forall(\mathbf{z}, \gamma) \in B_{\rho}\left(\mathbf{X}^{*}\right) \cap \mathcal{M}
$$

It is easy to verify that for the sequence $\left\{\left(\mathbf{z}_{*},(1-\right.\right.$ $\left.\left.\left.2^{-j}\right) \boldsymbol{\gamma}_{*}\right)\right\}_{j=1}^{j \rightarrow \infty}$, there exists an integer $N$, where $\left(\mathbf{z}_{*},(1-\right.$ $\left.\left.2^{-j}\right) \boldsymbol{\gamma}_{*}\right) \in B_{\rho}\left(\mathbf{X}^{*}\right) \cap \mathcal{M}, \forall j>N$ and $F\left(\mathbf{z}_{*},\left(1-2^{-j}\right) \boldsymbol{\gamma}_{*}\right)<$ $F\left(\mathbf{z}_{*}, \boldsymbol{\gamma}_{*}\right)$. This contradiction proves that Alg. 1 does not have a convergent point for 11 belonging to $\mathcal{M}$.

\section{Proof of Theorem 1}

Let $\left\{\mathbf{X}_{j}\right\}_{j=0}^{j \rightarrow \infty}$ be any arbitrary sequence generated by Alg. 1 for (14, which is due to Step 3 of Alg. 1, a decreasing sequence. By construction, we have formulated (14), so that the elements of $\left\{\mathbf{X}_{j}\right\}_{j=0}^{j \rightarrow \infty}$ will belong to a subset of the compact set $S^{n-1} \times[\varepsilon 1-\varepsilon]^{n-1}$.

We prove that $\left\{\mathbf{X}_{j}\right\}_{j=0}^{j \rightarrow \infty}$ is also a valid sequence for 13 , since it is a decreasing sequence for the objective function of 13, i.e., $F\left(\mathbf{X}_{j+1}\right) \leq F\left(\mathbf{X}_{j}\right), \forall k \geq 0$. Because $\left\{\mathbf{X}_{j}\right\}_{j=0}^{j \rightarrow \infty}$ is decreasing for (14), for this problem we have

$$
\begin{aligned}
& H\left(\mathbf{z}_{j+1}, \gamma_{j+1}\right) \leq H\left(\mathbf{z}_{j}, \gamma_{j}\right) \Rightarrow \\
& F\left(\mathbf{z}_{j+1}, \gamma_{j+1}\right)-v \sum_{i} \log \left(\gamma_{i_{j+1}}-\varepsilon\right) \leq \\
& \quad F\left(\mathbf{z}_{j}, \gamma_{j}\right)-v \sum_{i} \log \left(\gamma_{i_{j}}-\varepsilon\right) \Rightarrow \\
& \quad F\left(\mathbf{z}_{j+1}, \gamma_{j+1}\right)-F\left(\mathbf{z}_{j}, \gamma_{j}\right) \leq \\
& \quad v\left(\sum_{i} \log \left(\gamma_{i_{j+1}}-\varepsilon\right)-\sum_{i} \log \left(\gamma_{i_{j}}-\varepsilon\right)\right) \simeq 0 .
\end{aligned}
$$

From (16), we see that for an infinitesimal value of $v$, a decreasing sequence for (14) is also a decreasing sequence for (13). This means that unlike (14), for (13) we can consider the sequence $\left\{\mathbf{X}_{j}\right\}_{j=0}^{j \rightarrow \infty}$ belonging to the compact set $S^{n-1} \times[\varepsilon 1-\varepsilon]^{n-1}$. This is because unlike $(14), \gamma_{i}=\varepsilon$ belongs to the feasible set of $(13)$.

It is known that for a compact set any sequence owns a convergent subsequence [23]. Consequently, $\left\{\mathbf{X}_{j}\right\}_{j=0}^{j \rightarrow \infty}$ will have a convergent point, namely $\mathbf{X}_{*}$. By using Theorem 4.3.1 of [21]; which states every convergent point of a sequence generated by Alg. 1 is a critical point, we conclude the convergence of Alg. 1.

\section{REFERENCES}

[1] F. Barahona, M. Grötschel, M. Jünger, and G. Reinelt, "An application of combinatorial optimization to statistical physics and circuit layout design," Operations Research, vol. 36, no. 3, pp. 493-513, 1988.

[2] V. Cohen-Addad, V. Kanade, F. Mallmann-Trenn, and C. Mathieu, "Hierarchical clustering: Objective functions and algorithms," Journal of the ACM (JACM), vol. 66, no. 4, pp. 1-42, 2019.

[3] Y. Zhou, Y. Wang, P. Tang, S. Bai, W. Shen, E. Fishman, and A. Yuille, "Semi-supervised 3d abdominal multi-organ segmentation via deep multi-planar co-training," in 2019 IEEE Winter Conference on Applications of Computer Vision (WACV), pp. 121-140, 2019.

[4] M. M. Mohades, S. Majidian, and M. H. Kahaei, "Haplotype assembly using manifold optimization and error correction mechanism," IEEE Signal Processing Letters, vol. 26, pp. 868-872, June 2019.

[5] H. Shen and H. Zhang, "Improving exact algorithms for max-2-sat," Annals of Mathematics and Artificial Intelligence, vol. 44, no. 4, pp. 419-436, 2005.
[6] S. Sahni and T. Gonzalez, "P-complete approximation problems," Journal of the ACM (JACM), vol. 23, no. 3, pp. 555-565, 1976.

[7] T. Hofmeister and H. Lefmann, "A combinatorial design approach to maxcut," in Annual Symposium on Theoretical Aspects of Computer Science, pp. 439-452, Springer, 1996.

[8] M. X. Goemans and D. P. Williamson, "Improved approximation algorithms for maximum cut and satisfiability problems using semidefinite programming," Journal of the ACM (JACM), vol. 42, no. 6, pp. 11151145,1995

[9] S. Khot, G. Kindler, E. Mossel, and R. ODonnell, "Optimal inapproximability results for max-cut and other 2-variable csps?," SIAM Journal on Computing, vol. 37, no. 1, pp. 319-357, 2007.

[10] S. Khot, "On the power of unique 2-prover 1-round games," in Proceedings of the thiry-fourth annual ACM symposium on Theory of computing, pp. 767-775, ACM, 2002.

[11] J. Håstad, "Some optimal inapproximability results," Journal of the ACM (JACM), vol. 48, no. 4, pp. 798-859, 2001.

[12] W. Gosti, G. Nguyen, M. Wan, and M. Zhou, "Approximation algorithms for the max-cut problem," URL: http://citeseer. ist. psu. edu/489604. html, 1995.

[13] O. Dolezal, T. Hofmeister, and H. Lefmann, "A comparison of approximation algorithms for the maxcut-problem," in Manuscript, Universität Dortmund, Lehrstuhl Informatik, Citeseer, 1999.

[14] S. Burer, R. D. Monteiro, and Y. Zhang, "Rank-two relaxation heuristics for max-cut and other binary quadratic programs," SIAM Journal on Optimization, vol. 12, no. 2, pp. 503-521, 2002.

[15] P. Festa, P. M. Pardalos, M. G. Resende, and C. C. Ribeiro, "Randomized heuristics for the max-cut problem," Optimization methods and software, vol. 17, no. 6, pp. 1033-1058, 2002.

[16] P. Hansen and N. Mladenović, "Developments of variable neighborhood search," in Essays and surveys in metaheuristics, pp. 415-439, Springer, 2002.

[17] F. Glover, "Tabu search and adaptive memory programmingadvances, applications and challenges," in Interfaces in computer science and operations research, pp. 1-75, Springer, 1997.

[18] R. Martí, A. Duarte, and M. Laguna, "Advanced scatter search for the max-cut problem," INFORMS Journal on Computing, vol. 21, no. 1, pp. 26-38, 2009.

[19] M. Laguna and R. Marti, Scatter search: methodology and implementations in C, vol. 24. Springer Science \& Business Media, 2012.

[20] W. Zhu, G. Lin, and M. M. Ali, "Max-k-cut by the discrete dynamic convexized method," INFORMS Journal on Computing, vol. 25, no. 1, pp. 27-40, 2013.

[21] P.-A. Absil, R. Mahony, and R. Sepulchre, Optimization algorithms on matrix manifolds. Princeton University Press, 2009.

[22] C. Helmberg and F. Rendl, "A spectral bundle method for semidefinite programming," SIAM Journal on Optimization, vol. 10, no. 3, pp. 673696, 2000.

[23] V. I. Bogachev and O. G. Smolyanov, Real and functional analysis, vol. 4. Springer, 2020. 